\title{
ESTUDO COMPARATIVO DA DENSIDADE RADIOGRÁFICA DE CIMENTOS RESINOSOS
}

\author{
COMPARATIVE STUDY OF THE RADIOGRAPHIC DENSITY OF RESINOUS CEMENTS
}

\begin{abstract}
Ariadne de Araujo Goulart Pasquali *
Mauricio Rufaiel Matson "

Ricardo Raitz

\section{RESUMO}

Introdução: A radiopacidade dos cimentos resinosos revela a presença de partículas densas, sendo útil na identificação da adaptação de peças cimentadas. Nosso objetivo é comparar a densidade óptica de diferentes cimentos resinosos pelos métodos radiográficos convencional e digital. Métodos: Para cada cimento escolhido ( $\mathrm{C} \& \mathrm{~B}$, BisCem, Enforce) e para o grupo-controle (amálgama) foram confeccionados 5 corpos de prova (cp) idênticos em uma matriz de pvc. Cada um dos cp foi radiografado 3 vezes tanto pelo método convencional (utilizando-se filme periapical e aparelho de raios X intra-oral), quanto pelo digital (utilizando-se placas sensoras periapicais do sistema Digora-Soredex). As radiografias convencionais foram digitalizadas e a análise óptica da densidade dos materiais foi comparada pelo software Image J (NIH Image - Machintoch). Para o tratamento estatístico realizado pela ANOVA com 2 fatores e o teste complementar de Tukey com nível de significância de 5\%, os valores considerados foram os obtidos a partir da média dos valores entre as diferentes radiografias e cp. Resultados: os valores de densidade pelo método convencional e digital foram respectivamente para cada material: 232,87 e 255 para o amálgama; 136,73 e 136,24 para o Enforce; 135,44 e 148,51 para o Bis-Cem e 125,24 e 58,97 para o C\&B. Conclusöes: Não houve diferenças significantes entre os métodos radiográficos $(\mathrm{p}=0,069 \%)$. Foram verificadas diferenças significantes entre os materiais $(\mathrm{p}=0,00)$, em ambos os métodos, exceto entre o Enforce e o BisCem, que obtiveram média de tons de cinza semelhantes. $\mathrm{O}$ cimento $\mathrm{C} \& \mathrm{~B}$ mostrou as menores médias de tons de cinza.
\end{abstract}

DESCRITORES: Resinas compostas • Cimentos dentários • Radiografia digital dentária

\section{ABSTRACT}

Introduction: The radiopacity of the resinous cements reveals the presence of thick particles that are useful on observation of restorative adaptation. The aim of this study is to compare the optical density of different resinous cements, by using the conventional and the digital radiography. Methods: 5 samples (cp) of each resinous cements (C\&B, BisCem, Enforce) and for amalgam were identically built on a matrix of pvc. Each cp was radiographed 3 times both by the conventional method (using periapical film, as well as an intra-oral x-ray machine) and by the digital method (using periapical phosphor sensors plates of the Digora Systems, Soredex). The conventional radiographies were digitalized and the optical density of the studied materials was compared using the Image J software (NIH Image - Machintoch). ANOVA with 2 factors and Tukey test with level of significance of 5\% were used for statistical analysis and the considered values were those obtained from the average value of the different radiographies and cp. Results: The density values for the conventional and digital methods were, respectively, for each material: 232,87 and 255 for amalgam; 136,73 and 136,24 for Enforce; 135,44 and 148,51 for Bis-Cem and 125,24 and 58,97 for C\&B. Conclusions: There were no significant differences between the radiographic methods ( $\mathrm{p}=0,069 \%)$. Significant differences were found among the materials $(\mathrm{p}=0,00)$ in both methods, except for Enforce and for BisCem that presented similar histogram values. The cement C\&B showed the lowest average values of gray tones.

DESCRIPTORS: Composite resins $\bullet$ Dental cements $\bullet$ Radiography, dental, digial

\footnotetext{
* Aluna de graduação em Odontologia da Universidade Ibirapuera

** Mestre em Clínica Integrada e doutor em Dentística pela Faculdade de Odontologia da Universidade de São Paulo (FOUSP).

*** Mestre em Patologia Bucal e doutor em Diagnóstico Bucal pela Faculdade de Odontologia da Universidade de São Paulo (FOUSP); professor titular da Universidade Municipal de São Caetano do Sul e professor do Programa de Pós-Graduação em Radiologia do Instituto São Leopoldo Mandic..
} 


\section{INTRODUÇÃO}

A radiografia é um exame complementar em Odontologia, não apenas como auxiliar no diagnóstico, mas também para o entendimento das propriedades de materiais restauradores. A radiopacidade dos cimentos resinosos revela a presença de partículas densas e é uma propriedade requisitada para a maioria dos materiais restauradores dentários. ( $\operatorname{Cook}^{6}$ 1981, Farman et al. ${ }^{8} 1996$, Gu et al. ${ }^{12}$ 200Curtis et al. 1990 , Sidhu et al. ${ }^{18}$ 1996, Stanford et al. ${ }^{19}$ 1998, Curtis et al. ${ }^{7}$ 1990). Nos cimentos resinosos, visa à verificação de falhas na cimentação e a presença de infiltração marginal ocasionada pela cárie (Arkeboon et al. ${ }^{3}$ 1993, Cook $^{6}$ 1981).

No final da década de 80 iniciaram-se os estudos com a abordagem da imagem radiográfica digital na radiologia odontológica com softwares capazes de fazerem análises comparativas com maior facilidade e rapidez (Ferreira e Vale ${ }^{9}$ 1999). A digitalização de uma radiografia convencional introduz muitos elementos no processo diagnóstico, pois permite a melhora da imagem inicial através da manipulação matemática, interferindo no brilho e contraste e sua densidade. Assim, o manuseio da imagem proporciona uma melhora na percepção aos olhos humanos (Arkeboon et al. ${ }^{3}$ 1993, Bouschlicher et al. ${ }^{5}$ 1999, Vandre e Webber $\left.{ }^{20} 1995\right)$ favorecendo o diagnóstico. (Ohki et al. ${ }^{15}$ 1994, Raitz et al. ${ }^{16}$ 2006).

Por meio desses softwares é possível determinar também os níveis de cinza de uma imagem num histograma cujos valores variam de 0 a 256 . As imagens podem ser analisadas quanto ao histograma de tons de cinza determinando-se diferenças sutis de radiopacidade entre diferentes materiais (Cook ${ }^{6}$ 1981, Curtis et al. ${ }^{7}$ 1990).

O objetivo neste trabalho é comparar o grau de radiopacidade de diferentes cimentos resinosos disponíveis no mercado por meio da análise da densidade óptica em radiografias convencionais e digitais.

\section{MATERIAL E MÉTODOS}

Foram confeccionados 15 (quinze) anéis de PVC preto com $6 \mathrm{~mm}$ de diâmetro por $1 \mathrm{~mm}$ de espessura, com orifício no centro de $4 \mathrm{~mm}$ de diâmetro. Esses anéis, denominados de corpos de prova, foram preenchidos com cimentos resinosos fotoativados por 40 segundos para os cimentos duais. Para o cimento autopolimerizado, o tempo de fotoativação foi o preconizado pelo fabricante.

Cinco corpos de prova $(c p)$ para cada um dos cimentos relacionados foram confeccionados: Biscen (Bisco-
Illinois, USA); C\&B Cement Natural com Flúor Kit (Bisco- Illinois, USA); Enforce (Dentsply, USA). Sobre eles foi colocada uma placa de vidro para manter a superfície uniforme. $\mathrm{O}$ amálgama foi utilizado como controle radiopaco.

Cada $c p$ foi radiografado 3 vezes tanto pelo método convencional quanto pelo digital. No método digital os $c p$ foram colocados sobre a placa de imagem (sensor) do sistema digital Digora (Soredex, Milwaukee, USA). Para o método convencional, os $c p$ foram colocados sobre os filmes radiográficos periapicais da Kodak (E Speed). A exposição radiográfica em ambos os métodos se deu por meio de aparelho de Rx periapical da Astex, 70Kvp, com distância focal de $20 \mathrm{~cm}$. O tempo de exposiçẫo foi 0,8 e $0,4 \mathrm{~s}$, respectivamente para os métodos convencional e digital. Tanto os sensores quanto os filmes foram dispostos em uma mesa que os mantiveram em paralelismo com o cone.

Entre uma exposição e outra, apenas o sensor ou filme com o corpo de prova foi substituído, padronizandose a angulação. Posteriormente, as placas sensibilizadas (Digora) foram introduzidas na leitora óptica a laser do sistema para processar as imagens e os filmes foram processados em processadora automática.

As radiografias convencionais foram digitalizadas por um scanner de mesa com tampa de leitura de transparência com adaptador de slide e negativos da marca Microtek, modelo Scan Maker i800 (Microtek Lab, Carson, CA, USA), com 9600x4800 dpi de resolução, permitindo que a análise óptica da densidade dos materiais, em ambos os métodos, fosse realizada pelo software Image J (NIH Image - Machintoch). Por meio dele, verificou-se as médias de tons de cinza de cada material. Foi padronizada uma área circular de análise de 35 x 35 pixels onde foram analisadas a densidade máxima, mínima, média e seus respectivos desvios padrão.

Todos os dados foram tabulados e verificou-se não existirem diferenças estatísticas dos valores obtidos para as diferentes radiografias e $\mathrm{cp}$. Para o tratamento estatístico, os valores considerados foram aqueles obtidos a partir da média dos valores obtidos entre as diferentes radiografias e $c p$. Para tanto foi realizada a ANOVA com 2 fatores e teste complementar de Tukey com nível de significância de 5 .

\section{RESULTADOS}

O resultado da análise estatística encontra-se expresso na figura $\mathbf{1}$, em que letras diferentes significam 
diferenças estatisticamente significantes. Na figura 1 é possível verificar sensibilidades diferentes para os métodos estudados (convencional e digital) apenas para o cimento $\mathrm{C} \& \mathrm{~B}$.

Pelo método convencional, o amálgama obteve os maiores resultados de radiopacidade $(232,87)$, seguido pelo Enforce $(136,73)$ e Bis-Cem $(135,44)$, ficando o $\mathrm{C} \& \mathrm{~B}$ com as menores médias de tons de cinza $(125,24)$. No método digital as diferenças estatisticamente significante se mantiveram, com os valores de 255 para o amálgama; 136,24 para o Enforce; 148,51 para o Bis-Cem e 58,97 para o $\mathrm{C} \& \mathrm{~B}$.

\section{DISCUSSÃO}

A radiopacidade é uma propriedade importante dos materiais restauradores dentários (Am Dent Assoc ${ }^{1}$ 1981, Arkeboon et al. ${ }^{3}$ 1993, Berti et al. ${ }^{4} 2005$, Bouschlicher et al. ${ }^{5}$ 1999, Cook $^{6}$ 1981, Ferreira e Vale 1999 , Gonzalez e Woods ${ }^{10}$ 1993, Marouf e Sidhu ${ }^{14}$ 1998, Sabbagh et al. ${ }^{17}$ 2004, Sidhu et al. ${ }^{18}$ 1996), e nos cimentos resinosos visa à identificação de falhas na cimentação e infiltração marginal nas lesóes cariosas. (Am Dent Assoc ${ }^{1} 1981$, Cook $^{6}$ 1981, Curtis et al. ${ }^{7}$ 1990, Ferreira e Vale9 1999, Goshima $^{11}$ 1990). O conhecimento dessa característica e de outras propriedades como biocompatibilidade, adesão e estética, ajuda o profissional na escolha do material mais adequado. Neste estudo, tanto o cimento Enforce como o Bis-Cem apresentaram alta densidade óptica e que pode ser traduzida radiograficamente como maior radiopacidade. Além disso, não foram encontradas diferenças estatísticas entre ambos os cimentos. $\mathrm{O}$ cimento $\mathrm{C} \& \mathrm{~B}$ apresentou diferença estatisticamente significante em relação aos outros, apresentando os menores valores de tons de cinza em ambos os métodos radiográficos. Arkeboon et al. ${ }^{3}$ (1993), chamam a atenção para o fato de que, se materiais com diferenças substanciais em radiopacidade são usados em aplicaçôes combinadas para tratamento restaurador, a baixa radiopacidade pode interferir no diagnóstico de cárie ou detecção de fendas sob a restauração.

Os resultados encontrados também indicam que o método digital para o cimento $\mathrm{C} \& \mathrm{~B}$ mostrou menores valores de densidade óptica, o que pode interferir em uma análise clínica da qualidade de cimentação de trabalhos indiretos. Sendo assim, nesses casos, as radiografias convencionais podem ser preferíveis. Sabbagh et al. ${ }^{17}$ (2004), apesar de terem encontrado uma correlação linear entre o método radiográfico convencional e o método digital pelo sistema Digora, afirmaram que o método convencional parece ser mais preciso para as mensuraçôes de radiopacidade, confirmando os resultados deste trabalho.

Rotineiramente, utiliza-se o fotodensitômetro para a leitura de densidade em filmes radiográficos de acordo

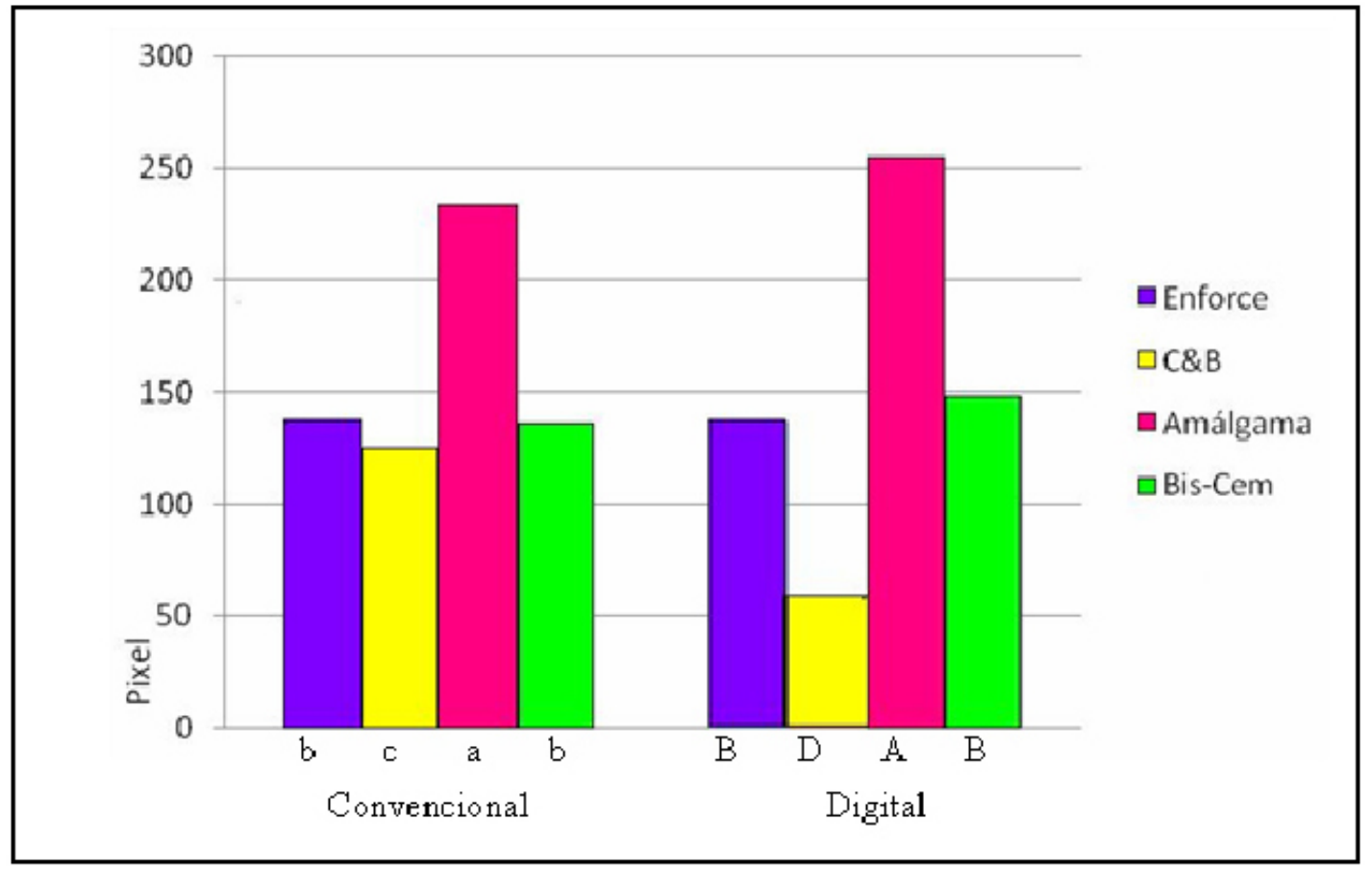

Figura 1- Gráfico da análise dos tons de cinza dos corposde-prova. Letras diferentes significam diferenças significantes ao nivel de 5\%. 
Pasquali AAG, Matson MR, Raitz R. Estudo comparativo da densidade radiográfica de cimentos resinosos. Revista de Odontologia da Universidade Cidade de São Paulo 2009 set-dez; 21(3): 239-43

com as especificaçóes da American Dental Association. (Am Dent Assoc ${ }^{1}$ 1981, Berti et al. ${ }^{4}$ 2005, Stanford et al. ${ }^{19}$ 1987). Muitas vezes, a radiopacidade de um material é comparada à radiopacidade de um dispositivo com degraus de alumínio (penetrômetro), que se convencionou chamar de milímetros de alumínio (American National Standars ${ }^{2}$ 1984). Neste trabalho, a densidade óptica foi medida por meio da análise do histograma de tons de cinza que varia de $0-256^{10}$, podendo traduzir radiograficamente a radiopacidade (Cook ${ }^{6} 1981$, Curtis et al. ${ }^{7}$ 1990). Os computadores tornaram-se ferramentas indispensáveis na rotina do profissional de saúde, pois, cada vez mais, disponibilizam-se softwares que oferecem inúmeras facilidades relacionadas ao processamento da imagem, morfologia e reconhecimento das estruturas e do próprio sistema de análise de imagens baseados no conhecimento (Gonzalez e Woods ${ }^{10}$ 1993). Esse novo paradigma veio para modificar radicalmente a maneira como o clínico vê e interpreta uma imagem, pois permite a manipulação de algoritmos matemáticos interferindo no brilho, contraste, e densidade da imagem, adequando-a para cada observador. (Kullendorf et al. ${ }^{13}$
1996, Ohki et al. ${ }^{15}$ 1994, Raitz et al. ${ }^{16}$ 2006, Vandre e Webber ${ }^{20} 1995$ ).

A imagem digital direta permite ainda a avaliação em tempo real e diminui erros de processamento (Curtis et al..$^{1990 \text {, Ferreira e Vale } 9}$ 1999, Gonzalez e Woods ${ }^{10}$ 1993, Goshima ${ }^{11}$ 1990, Kullendorf et al. ${ }^{13}$ 1996, Ohki et al. ${ }^{15}$ 1994). Todas essas vantagens podem ser interessantes para métodos de diagnósticos de doenças bucodentais, mas, conforme os resultados observados, utilizando-se radiografias digitais para análise da qualidade de cimentação de trabalhos indiretos, sua indicação pode ser questionada.

\section{CONCLUSÃO}

Conclui-se que houve diferença estatisticamente significante entre os métodos radiográficos apenas para o cimento $\mathrm{C} \& \mathrm{~B}$. Em relação aos cimentos, foram verificadas diferenças significantes em ambos os métodos, exceto entre o Enforce e Biscem, que obtiveram médias de tons de cinzas semelhantes. $\mathrm{O}$ cimento $\mathrm{C} \& \mathrm{~B}$ apresentou os menores valores de densidade óptica.

\section{REFERÊNCIAS}

1. American Dental Association Council On Dental Materials, Instruments And Equipments.The desirability of using radiopaque plastic in dentistry: a status report. J Am Dental Assoc 1981;102(3):347-9.

2. American National Standards Institute. Specification n 57 for endodontic filling materials. J Amer Dent Ass 1984;108:88.

3. Arkeboon HBM, Kreulen CM, Van Amerongen WE, Mol A. Radiopacity of Posterior Composite Resins, Composite Resin Lutin Cements, And Glass Ionomer Lining Cements. J Prosth Dent 1993;70(4):351-5.

4. Berti, SA, Souza PHC, Westphalen FH, Westphalen VPD, Martins WD, Ignácio SA. Estudo radiográfico da densidade óssea mandibular em pixels e milímetros equivalentes de alumínio. Revista Odonto Ciência 2005;20(49):251-256.

5. Bouschlicher MR, Cobb DS, Boyer DB. Radiopacity of compomers, flowable and convencional resin composites for posterior restorations. Oper Dent 1999;24(1):20-5.
6. Cook WD. An investigation of radiopacity of composite restorative materials. Aust Dent J 1981;26(2):105-12.

7. Curtis PM Jr, von Fraunhofer JA, Farman AG. The radiographic density of composite restorative resins. Oral Surg Oral Med Oral Pathol 1990;70(2):226-30.

8. Farman TT, Farman AG, Scarfe WC, Goldsmith LJ. Optical densities of dental resin composite: a comparison of CCD, strage phosphor, and ektasped plus radiografic film. Gen Dent 1996;44(6):532-7.

9. Ferreira FBA, Vale MS, Tavano O. Radiopacidade de cimentos endodônticos avaliados pelo sistema de radiografia digital. Rev FOB 1999; 7(1-2):55-60.

10. Gonzalez RC, Woods RE. Digital Image Processing. New York: Wesley; 1993.

11. Goshima T, Goshima Y Radiographic detection of recurrent carious lesions associated with composite restorations. Oral Surg Oral Med Oral Pathol 1990;70(2):263-9. 
12. Gu S, Rasimick BJ, Deusch AS, Musikant BL. Measuring the radiopacity of luting cements, dowels, and core build-up material with a digital radiography system using a CCD sensor. J Prosthodont 2007; Set-Oct 16(5): 357-64.

13. Kullendorf B, Nilsson M, Rohlin M. Diagnostic accuracy of direct digital dental radiograph for the detection of periapical bone lesions. Overall comparison between conventional and direct digital radiography. Oral Surg Oral Med oral pathol Oral Radiolg Endod 1996;82(3): 344-50.

14. Marouf N, Sidhu SK. A study on the radiopacity of different shades of resin-modified glass-ionomer restorative materials. Oper Dent 1998;23(1):10-4.

15. Ohki M, Okano T, Nakamura T.Factors determining the diagnostic accuracy of digitizd conventional intraoral radiographs. Dentomaxillofac Radiol 1994;23:77-82.
16. Raitz R, Curi M, Dib L, Fenyo-Pereira M. Conventional and indirect digital radiograph interpretations or oral unilocular radiolucent lesions. Dentomaxillofac Radiol 2006;35(3):165-9.

17. Sabbagh J, Vreven J, Leloup G Radiopacity of resin- based materials measured in film radiographs and storage phosphor plate (Digora). Oper Dent 2004;29(6):677-84.

18. Sidhu SK, Shah PMM, Chong BS, Pitt Ford TR. Radiopacity of Resin Modified Glass-ionomer Restorative Cements. Quintessence Int 1996;27:639-4.

19. Standford CM, Fan PL, Schoenfeld CM, Knoeppel R, Standford JW Radiopacity of Light-cured posterior composite resins. J Am Dent Assoc 1987;115(5):722-4.

20. Vandre RH, Webber RL. Future trends in dental radiology. Oral Surg Oral Med Oral Pathol Radiol Endod 1995;80(4):471-8.

Recebido em: 13/7/2009

Aceito em: 1/10/2009 